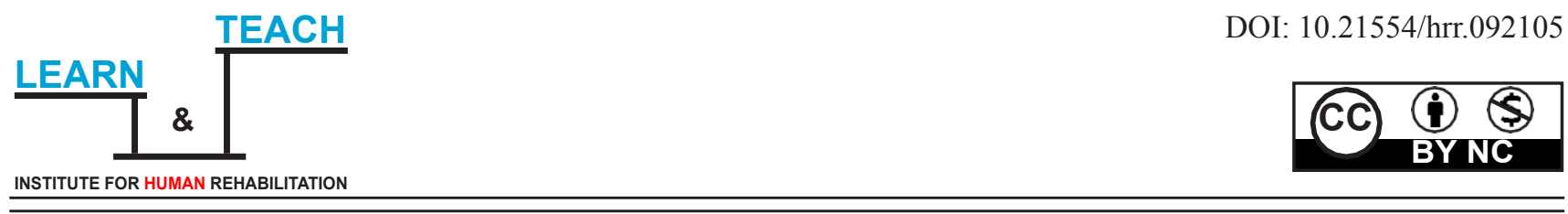

\title{
PSYCHODRAMA - THE METHOD WHICH PRODUCE CHANGE IN THE QUALITY OF LIFE OF PSYCHOTIC PATIENTS
}

\author{
Jasna Veljkovic ${ }^{1}$, Dragomir Davidovic ${ }^{2}$, Ivana Leposavic ${ }^{3}$, Maja Davidovic ${ }^{2}$
}

Original scientific paper

${ }^{1}$ Faculty of Political Sciences, University of Belgrade, Republic of Serbia

${ }^{2}$ Clinic for psychiatric diseases 'Dr Laza Lazarevic", Belgrade, Republic of Serbia

${ }^{3}$ University Clinic for Psychiatry, Belgrade School of Medicine, Republic of Serbia

Received: $2021 / 5 / 13$

Accepted: 2021/8/9

\begin{abstract}
The aim of this a quasi-experimental study is to test the changes in the Quality of Life of chronic mental disorders (schizophrenia) treated with psychodrama. Total sample consisted of 60 subjects, aged 21-38. From these, 30 subjects were in the experimental group, while 30 subjects were in the control group. All subjects were previously psychologically tested, as well as basic demographic data has been taken. The instrument we used was Life Quality Scale in Schizophrenia (21 items form). Qualitative and quantitative analysis of the obtained data indicates a statistically significant improvement of social and communicative abilities of participants of experimental groups, better impulse control and activation in the work area. The disadvantage of this research is: the small sample. Future examination is needed, as well as a more advanced data processing methodology.
\end{abstract}

Keywords: gender, group psychotherapy, life quality, psychodrama, psychotic patients, schizophrenia

\section{INTRODUCTION}

„Fear of breakdown is the fear of breakdown that has already been experienced. " $D$. W. Winnicott

Moreno's psychodrama approach opens a new model of thinking about human beings in relation to themselves and others. The findings of the application of this method indicate that psychodrama has a special role in the development and integration of the self (Moreno, 1946; Veljkovic, 2015). Unlike Freud, who believed that psychotic disorders could not be treated with psychotherapy, Moreno believed that the fact that someone suffers from schizophrenia or schizophrenic disorder does not exclude the possibility of psychotherapeutic treatment (Veljkovic, 2014). Moreno had also used psychodrama in the treatment with acute psychotic patients in the 1940s at his Baecon Sanatorium in New York. He believed that the longer a psychotic experience stays within a person, the more dangerous it becomes for his functioning, but also for other people who are in his immediate vicinity (Veljkovic, 2019).
His Psychodrama principle consists of providing meaning for the objectification of the order of inner reality. Recent world research indicates the effectiveness of the application of psychodrama in the treatment of many psychopathological categories. Comparison between the experimental and control group of female subjects who suffered from psychotic depression, showed significant differences, after the psychodrama treatment, measured by covariance analyses. Conclusion was that psychodrama leads to decrease of depression at intervention group (Bellil, 2010).

The evaluation studies applied to the different diagnostic categories gave findings that psychodrama could produce good therapeutic effect in several categories of mental disorders This are the next categories: Mental and behavioral disorders due to psychoactive substance abuse (ICD-10, F.10-F.19); Schizophrenia, schizotypal and delusional disorders ( ICD-10, F. 20.-F. 29); Mood disorders (ICD-10,F.30-F39), Behavioral emotional disorders with onset usually in childhood or adolescence (ICD-10,F.40-F.48);

\footnotetext{
Correspondence to:

Jasna Veljkovic, Faculty of Political Sciences, University of Belgrade, Republic of Serbia

Belgrade, Republic of Serbia

E-mail: jasnapsi@gmail.com
} 
neurotic, stress related and somatoform disorders (ICD 10, F. 30-F.39). The main objection of these studies is more than naturalistic approach (Wieser, 2007). The analysis of studies on treatment effects of the psychodrama therapy should be carried out on the background of the ICD-X to identify the constraints of research encountered in this field and suggest us possible avenues for future research. We shall present a kind of statistic evidence that we have for psychodrama effectiveness, according to ICD -X diagnostic criteria of mental disorders. This research refers to the examination of changes in the Life Quality in a patient with psychotic disorders who were on psychodrama treatment.

Subject and objective of the study:

The subject of the present study is to establish:

a. to what extent the global quality of life of subjects with recurrent psychotic episodes in the experimental and control groups is impaired;

b. changes in global quality of life between the subjects of the experimental and control groups "before and after" one year;

a. differences in the quality of life of subjects with psychotic disorders in relation to different variables (sex, education, work activity, number of readmissions, day functionality, etc.);

b. the influence of the duration of group therapy (in years) in the experimental group of subjects, - on changes in the quality of life.

Objective of the research was to investigate the connection between certain socio-demographic parameters and the quality of life. Our goal was to verify existence of differences in quality of life between the experimental and control groups, taking into account the significance of psychodrama treatment for the treatment outcome. Also, we expected better quality of life in subjects which spent longer time on psychodrama group treatment.

\section{METHODOLOGY}

\section{Sample}

The sample consisted of total 60 respondents in clinical population and were divided into two following groups:

1. Experimental $(30$ subjects with psychotic disorders, who were treated by psychopharmacological treatment and also with psychodrama group treatment, once per week.

2. Control group (30 subjects with psychotic symptoms, who were treated only by psychopharmacological treatment.

Both groups of patients were equalized according to certain criteria and the criteria were: gender, age, educational level, marital status, diagnoses according to ICD-10, criteria/F20-F29/. According to the gender representation in relation to the group to which they belong, statistically significantly more women were covered by psychodrama treatment.
The average age of patients in the experimental group was $31.90+5.02$ years, while in the control group it was $31.00+5.44$ years. The average height of IQ subjects in the experimental group was $113.53+6.49$ and in the control was $107.80+5.51$. All subjects were within the range of average intelligence. There is a significant difference in the average height of IQ subjects in the experimental group of subjects by sex, $\mathrm{F}=11.213, \mathrm{p}<.001$, in a a favor of woman.If the education of the subjects from the experimental group is taken into account, $40 \%$ have a university degree, while the higher education has $(43.33 \%)$. In the control group, the higher education $(20 \%)$, and the same number of subjects have university degree $(20 \%)$. Nonparametric correlation coefficient, indicates a statistically significant inverse correlation between groups, by education, for $\mathrm{p}<.001$. Statistically, there are significantly more subjects living with their parents, regardless of the group to which they belong. All subjects, both experimental and control groups, were treated were hospitalized than have outpatient treatmentin the Institute for Neuropsychiatric Diseases: "Dr. Laza Lazarevic". Prior to entering the examination procedure, all of them were psychologically tested and were on treatment with appropriate psychiatric drugs. Entry for treatment with psychodrama group therapy was preceded by a certain selection according to the relevant criteria. Contraindications were: currently psychotic, highly hostile, highly paranoid, suicidal and homicidal subjects. The criteria for entering the group were: expressed motivation of the subject to enter the group with previously demonstrated qualities for communication during the hospitalization, younger age of the respondents, at least secondary school, at least average IQ and 1 to 3 previous admissions in psychiatry hospital. Respondents who were not motivated for group therapy represented the control group. Our previous researches were with asmall samples of subjects, but they has shown the effectiveness of the psychodrama method especially if the treatment starts immediately, after the first admission (Veljkovic \& Djuric, 1997; Veljkovic, 2014). It is important to note that time interval between the test and re/test in the experimental and control group wasone year. After one year, the experimental group continued to exist, because of the high motivation the most of the group members. The members of experimental group has spent different time on psychodrama treatment, form $1-6$ years. We continued to monitor the qualitative changes in their life quality, until they decide tolive the group.

\section{Instruments}

In the Introductory part of this research, the standard psycho-diagnostic instruments were applied: clinical interview, Wechsler Intelligence Scale (Serbian standardization), andMinnesotaMultiphasicPersonality Inventory (MMPI/202, Serbian standardization). For the purposes of the presented research, we present the results of their Life quality on the QOLS scale. 
The World Health Organization (WHO, 1992) by quality of life means - the perception of an individual about their own position in life within the culture and values of the system in which they live, and in relation to the goals, expectations, standards and problems of the environment (The World Health Organization Quality of Life Assessment, 1998). ${ }^{1}$ The Quality of Life Scale in Schizophrenia (QLS), is an instrument for Rating Schizophrenic Deficit Syndrome (Heinrich, Hanlon, \& Carpenter, 1984), translated into Serbian language.The authors describe the Quality of Life Scale (QLS), a 21item scale based on a semi structured interview designed to assess deficit symptoms and thereby fill an important gap in the range of instruments now available.

\section{Data Processing}

Thecollecteddatawereprocessedapplyingmethodologycal of descriptive and interferential statitsics. The data are presented by dividing the frequency of respondents by categories of examined characteristics. In presenting the results of the research, frequency distribution tables were used as well as direct analysis of tabular data and analysis based on graphical representation. The conclusions and observed characteristics necessary for giving grades and conclusions were made.
Relationship indicators, i.e. statistical description, are presented by: a measure of the variability of a statistical series, intervals of variation, standard deviation and coefficients of variation. Data obtained through measurements are presented using central tendency measures.

Univariate methods were used to use the significance of the difference, namely: nonparametric tests and proportionality tests for attributive features such as the chi-square test and the proportionality test.

Parametric tests - ANOVA test and Student's T-test were used to test the significance of the difference in numerical features. In addition, multivariate statistical methods were used, as well as methods for testing parallelism. Statistical processing and analysis was done in SPSS ver.12.0, and graphical and tabular presentation was done in relevant statistical computer program.

\section{RESULTS}

The analysis of the average values of QLS, in the patients by groups, gave the following differences between the patients in relation to the group to which they belong, experimental or control. The results are as follows:

Table 1. The average value of the QLS scale in patients by groups

\begin{tabular}{clccccccc}
\hline \multirow{2}{*}{ QLS } & \multirow{2}{*}{ Groups } & \multirow{2}{*}{ N } & Average & SD & \multicolumn{2}{c}{$\mathbf{9 5 \%}$ Interval } & \multirow{2}{*}{ Min. } & Max. \\
\multirow{2}{*}{ QLS test } & Experimental & 30 & 42.63 & 6.12 & 40.35 & 44.92 & 32 & 55 \\
& Control & 30 & 40.05 & 2.82 & 38.73 & 41.37 & 36 & 45 \\
& Sum & 60 & 41.60 & 5.19 & 40.13 & 43.07 & 32 & 55 \\
\hline \multirow{2}{*}{ QLS retest } & Experimental & 30 & 67.87 & 7.65 & 65.01 & 70.72 & 49 & 78 \\
& Control & 30 & 46.25 & 3.54 & 44.59 & 47.91 & 41 & 53 \\
& Sum & 60 & 59.22 & 12.41 & 55.69 & 62.75 & 41 & 78 \\
\hline
\end{tabular}

In the experimental group, the average value of the QLS scale is statistically significantly higher on retesting compared to the same value of the QLS scale in the control group for $F$ test $=139.00$, for $p$ $<.001$. Analysis of the average values of the QLS scale in patients by groups gave the differences between patients in relation to gender: in the females of experimental group, the average value of the QOL was statistically significantly higher on the first test, $\mathrm{F}$ test $=7.391$, for $\mathrm{p}<.01$. In the control group, the average value of the QLS scale was higher in females but not statistically significant.

\footnotetext{
${ }^{1}$ Although there are papers in the world that give a critical review of the real flood of papers on quality of life (underlining the difficulties in applying research results and the ease of conducting research using questionnaires), in our country such research is still in the initial phase. Quality of life, besides significant influence of depressiveness, personality characteristics and adaptive mechanisms of diseased, can serve as minimal standard of treatment outcome indicators for schizophrenic patients. A significant indicator of treatment success could be improvement of subjective experience of the quality of life of patients with schizophrenia. Some authors concerns about using self-report data to measure "objectively" defined QOL (Lehman, 1998)."Despite limitations in the tools, QLS research has focused on three key areas: 1) Descriptive studies in which QOL of various populations of chronically mentally ill are measured and compared;2) Association studies in which patient characteristics are associated with QOL and 3) Intervention studies in which QUL is used as an outcome" (Veljkovic, 2011,:18). The construct of quality of life (QOL) has become important with the increasing emphasis on evaluating treatment outcomes and patient satisfaction with health care. The Quality of Life scale, created originally by American psychologist John Flangan in 1970's, was made for use in chronic illness groups. Reliability, content and cosntruct validity testing has been performed on the QOLS and had a number of translation. Quality of life measures have become more important for therapeutic interventions and clinical decisions, especially for patients with chronic illness. Therefore, the schizophrenia-specific QOL measures are particularly useful for understanding how the symptoms affect the live quality of those people. There are several versions of QOL scales in schizophrenia. We used 21.item scale, based on a semi structured interview with subjects, designed to assess deficit symptoms.
} 
On retesting in the experimental group, the values of QLS scale were statistically significantly higher, in female of experimentally group, $\mathrm{F}=9.321$, for $\mathrm{p}<.01$, but not in the control group. In the experimental group of mails, the value of the QLS scale increased statistically significantly on retesting after psychodrama treatment for the value of the Student's T test $=25.321$, for $\mathrm{p}<$ .001 , also in females for the value of Student's $\mathrm{T}$ test $=$ 28.741 , for $\mathrm{p}<.001$. In the control group according to sex, the average value of the QLS scale didnot increase statistically significantly on retesting compared to the values of the QLS scale on the first testing. The experimental group, by both genders, had statistically significantly higher QLS scale values on both testing and retest.

There was no statistically significant difference in the level of the values on the QLS scale in relation to the age of the experimentalgroup on the first test. The average values of the QLS scale were equal among subjects by age groups. There was no statistically significant difference in the height of the QLS scale compared to the age group in the control group.

The highest average values of the QLS scale in the experimental group, on retest had patients aged 20 to 29 years.On retestthere was a statistically significant difference in the level of the QLS scale in relation to the age in experimental group, for $\mathrm{F}=4.356$ for $\mathrm{p}<.02$. In the control group, there was no statistically significant difference in the level of the QLS scale in relation to the age. Life quality was statistically significantly improved in all the subjects of experimental group, and the greatest positive changes was in the age group of 20 to 29 years for $p<.001$.In the control group, there was no statistically significant difference in retest compared to the first test.

The influence of subjects education level on the life quality in experimental group, on the first test, didn't give a statistically significant differences; on retest, subjects rated their quality of life statistically significantly higher, where the largest increase in the life quality was in subjects with higher education, for $\mathrm{p}<.01$.

In the control group, on the first test there was not statistically significant difference in QLS scores, compared to educational level, average QLS values were uniform, and on retest, there was certain improvement, but not statistically significant, in relation to the values of the first testing.

Work activity was also tested in subjects of the experimental group; at the first test, we registered a statistically significant differences; average QLS scores were lowest in the unemployed subjects, $39.33+5.87$, and highest in students, $45.50+2.12$ for $F$ test $=2.781$ for $\mathrm{p}<.05$; on retest, subjects from experimental group, rated their work activity statistically significantly higher, where the largest shift was in unemployed subjects, for $\mathrm{p}<.001$, and then in employed subjects, for $\mathrm{p}<.01$, but also statistically significantly higher values on QLS for $\mathrm{p}<.01$.
In the control group of subjects, there was not statistically significant difference in QLS scores in relation to work activity; the average values of QLS were equal, and on retest, there was registered an improvement in the life work activity but not statistically significant in relation to the test values. Within the value of QLS on the retest there is only a statistically significant difference, in the students who rated their work activity significantly lower than employees on the retest for $\mathrm{F}=3.949$ for $\mathrm{p}<.03$.

The following differences were found between patients in relation to the number of hospitalizations. In the experimental group, the average value of the QLS was statistically significantly improved, where the average value of the QLS scale was inversely correlated with the number of hospitalizations, QLS declined with more frequent hospitalization for $F=4.669 \mathrm{p}<.01$. In the control group of subjects, the average value of the QLS scale analyzed by the number of hospitalizations was not statistically significantly different on the retest, where the average value of the QLS scale was directly correlated with the number of hospitalizations. If we observe the values of QLS scores of experimental group, that describe family relationships, social communication outside of work, then work functioning, degree of motivation and curiosity of patients, sexual life and ability of for empathy, testretest shows the following results: in subjects of experimental group there is significantly improvement of their relationships with family members and usually rate them as good, for $p<.05$; there is significantly better social communication of subjects, for $\mathrm{p}<.01$; the subjects has a higher score of sexual functioning, but not statistically significant for $\mathrm{p}=\mathrm{ns}$; statistically significantly better is working functioning for $\mathrm{p}<$ .05 ; statistically significantly is better perceiving their goal for $\mathrm{p}<.01$, motivation has also increased statistically significantly, $\mathrm{p}<.05$, and curiosity, for $p<.01$. The ability to empathize in the subjects of the experimental group was assessed as poor before entering the treatment. After one year on retesting, the assessment of the ability of subjects for empathy is statistically significantly higher, for $\mathrm{p}<.05$, and interaction with the examiner is better assessed, for $p$ $<.05$, assessment of daily functioning is statistically significantly higher as well as spending time and daily activities. The subjects of the control group showed some improvement in these areas of life but not statistically significant.

After one year from we continued to observe only the subjects of the experimental group, as that group continued its work. Further, we follow the results related to the quality of life of the subjects of the experimental group in relation to the length of time that they have spent on the group treatment. 
Table 2 The influence of the duration of group therapy (in years) on the average level of QLS in subjects of experimental group

\begin{tabular}{ccccccc}
\hline $\begin{array}{c}\text { Years spent on } \\
\text { psychodrama } \\
\text { treatment }\end{array}$ & $\begin{array}{c}\text { Number of } \\
\text { patients }\end{array}$ & $\begin{array}{c}\text { QLS } \\
\text { before }\end{array}$ & $\begin{array}{c}\text { QLS after } \\
\text { 1 year }\end{array}$ & $\begin{array}{c}\text { QLS } \\
\text { before }\end{array}$ & $\begin{array}{c}\text { QLS after 1 } \\
\text { year }\end{array}$ \\
\cline { 3 - 7 } & Average & Average & SD & SD & t \\
\hline $1-2$ & 5 & 50.00 & 70.60 & 4.90 & 4.67 & $\mathbf{- 6 . 8 0 6}$ \\
$2-3$ & 5 & 40.20 & 70.00 & 4.38 & $\mathbf{7 1}$ & $\mathbf{- 1 5 . 0 1 3}$ \\
$3-4$ & 4 & 46.25 & 69.25 & 3.40 & 10.31 & $\mathbf{- 4 . 2 3 8}$ \\
$4-5$ & 9 & 41.44 & 71.67 & 5.55 & 6.16 & $\mathbf{- 1 0 . 9 3 3}$ \\
$5-6$ & 7 & 38.57 & 58.71 & 5.03 & 5.82 & $\mathbf{- 6 . 9 2 7}$ \\
\hline
\end{tabular}

Subjects whose average value of the QLS scale after the first year of group treatment ranged from $69.25+$ 10.31 to $71.67+6.16$, spent from one to five years on group treatment. Five and more than five years on the group treatment spent subjects whose average value of the QLS scale after the first year of treatment was statistically significantly the lowest and amounted to $58.71+5.82$. The value of QLS in subjects who spent on the group psychotherapy from two to three years was highest $\mathrm{T}$ test $=-15.013, \mathrm{p}<.000$; and then in subjects who were treated in group for four to five years, $\mathrm{T}$ test $=-10.933$ for $\mathrm{p}<.001$.

\section{DISCUSSION}

The test-retest findings of the QLS scale clearly report the result that the quality of life significantly improved in the subjects of the experimental group. This is evidenced by a statistically significant finding on the retest of this group of subjects after the application of psychotherapy for the value of the Student's $\mathrm{T}$ test $=-14,441$, for $\mathrm{p}<.001$. In the control group of subjects, the quality of life improved but not statistically significantly. The average value of the QLS scale increased at retest, but not statistically significantly. This finding indicates that there was a statistically significant improvement in quality of life only in the experimental group of subjects, treated with psychiatric drugs and psychodrama method of group psychotherapy. This supports the fact that psychopharmacs reduce manifest psychotic symptoms but not the socio-psychological consequences of a process psychotic disorder (Herrman, Hawthorne, \& Thomas, 2002). Homogeneous groups whose members are schizophrenic patients are clinically more successful than heterogeneous groups (Barr, 1986). In the hospital environment, greater effectiveness of the group was observed through longer-term group treatment. Interaction-oriented groups proved to be more effective than those that were insight-oriented. In the spectrum of psychotic disorders, group therapy is indicated in patients with schizophrenia and similar psychotic disorders, as well as in bipolar disorder in the stable phase of the disease (Ruzic, Medved, DadicHero, \& Tomljanovic, 2009). According to Sullivan, psycho-social group treatment is very important for the better outcome of shizophrenia (Sullivan, 1974).
Plenty of research has confirmed that quality of life depends on general social support (Heideret al., 2007; Eack, Newhil, Andreson, \& Rotondi, 2007). The most positive effects on the quality of life of schizophrenic patients, as shown by numerous studies, produce integrative treatments of this category of subjects (Holzner, Kemmler, \& Meise, 1998; Schmidt, Staupendahl, \& Vollomoeller, 2004). The application of integrative treatment significantly improves the quality of life of patients with psychotic disorders.

On retest in the experimental group of subjects, the average value of the QLS scale are statistically significantly higher in women for $\mathrm{F}=9.321$, for $\mathrm{F}=$ 9.321 , for $\mathrm{p}<.01$, but not in females on the retest of the control group. From this follows a finding that females benefit more from treatment in the psychodrama group than male subjects; this fact can be discussed from a psychological and psychiatric aspect. If we use the data obtained from the practice of researchers, but which is not statistically verified, that is that women much more frequent are protagonist in psychodrama and are more active than man on the group. The results of some studies confirm that women have a less serious course of the psychotic illness disease compared to men, less number of admissions, more frequent outpatient treatment, less frequent occurrence of negative symptoms, better social adaptation, and a good therapeutic response to lower doses of drugs (Ochoa, Usall, Cobo, Labad, \& Kulkami, 2012).The psychiatric aspect of viewing would probably deal with the very genesis of process psychosis in men and women, and the fact is that the same diagnostic categories may have different manifestations and different course. According to Brkic et al, the incidence of the disorders that consist schizophrenia spectrum and also sex differences within the group are important to be evaluated.In their research on issue of schizophrenic spectrum and differences in representation in relation to gender found that in women, schizoaffective disorder was more often, while in male the same casewas with schizophrenia (Brkic, Tadic, Stojiljkovic, \& Maric, 2005). Further considerations may go in the direction of greater readiness of women to discover their own inner self, greater spontaneity and greater motivation for authentic change in terms of training for a more independent life in psychosis. 
Regardless of the age of most subjects in the experimental group, the quality of life at the beginning of the study was assessed as poor. An interesting finding is that after one year, there was a statistically significant difference of QLS scale values in relation to the age group to which they belong only in the subjects of the experimental group. The largest positive changes in the values of QLS scale scores were registered in the subjects of the experimental group aged 20 to 29 years; but in the control group, there was no statistically significant difference in QLS scores, on the test-retest according to age. The age at which process psychosis begins in men is atearlier age than, in women. Although the overall incidence is approximately the same in both sexes, the average age at which schizophrenia is diagnosed in men is 15-24 years, and 25-34 years in women. Early onset of the psychotic illness is associated with a more severe course and a worse prognosis compared to the later onset of the disease. In men, the first acute episode usually occurs after the age of 20 and in woman, after 25.

Most of patients on the experimental group accepted their psychotic illness, during the time which they spent on the group. Accepting themselves with their psychotic illness at the beginning usually leads them to resignation; later the group members share their fight with all the difficulties and they feel as they are fighting for the same thing, for the better living. The finding that the best result in the improvement of life quality was in female subjects of younger age, which suggests us that psychodrama group treatment should begin immediately, after the first psychotic decompensation. The optimal time is after they establishment of the first stable remission after the first onset of psychotic illness. Also, that we could expect better results of treatment in females than in male subjects, in the age of 20-29.

One finding clearly indicates that the educational level can be one of the predictors of the success of psychotherapeutic treatment in subjects with psychotic disorders is interesting, but this is not the most important predictor.Also, in the subjects of the control group were improvements in the quality of life in relation to the educational level, but not statistically significant. There are not the studies which could support our findings. But thisfinding indicates us, that subjects with psychotic disorders (control group) who are on the higher educationalduring the progression of psychosis, gradually stop using their knowledges. Our free interpretation refers to the fact that the cause of this is not only a psychotic process with its known characteristics, but also the lack of intellectual stimulation that the group situation encourages. Their educational qualities remain inactive and unused for the purpose of their further progress, which does not happen with subjects who are on group therapy. Group therapist and the member of the group are permanently required from subjects to recognize and activate their own qualities and knowledges.

If we consider the work activity of the subjects of the experimental group in relation to quality of life, the largest increase on the QLS scale was in unemployed subjects, then in employees, and the smallest improvement in quality of life had students, but also statistically significantly higher. In the subjects of the control group - in relation to the work activity on retest, there was an improvement but not statistically significant in relation to the values of the first test. For unemployed subjects of the experimental group at the beginning of group treatment, coming on the group was often the only way to go out of the house. The group remains their only social microcosmos for a long time. The group from a group analytical point of view, become for them as a "good mother" (Foulkes, 1984), and stimulate them to find any job and to work. Many group members found some part -time job, in order to be gratified from the group. The biggest impact of group work on the unemployed subjects is that the group in the beginning is the only social environment for them and "awakes them from a long dream" (Rosenfeld, 1965); during the time subjects begin to trust the group as a whole and accept its influence; after some longer time, positive transference happen, and they become less resistant to the other social influences; with increasing communicativeness in the group, their interactive sociability grow in society in general and their work ability improves.

Employed subjects of the experimental group have a slightly smaller improvement in their work results than the unemployed, but still a statistically significant improvement. This data is interesting in relation to the group of students who have the smallest increase in results in improving the quality of life in relation to the unemployed and employed.This data contradicts the previous data related to the age category, which is that in the category of 20-29 years, the best results are in the field of quality of life. The apparent contradiction is that students in Serbia are unfortunately often considered to be those whose age is older than 25 years. A detailed analysis of statistical data shows that in this case we are talking about "eternal" students, because most of them enrolled in the same year more than once, so in fact they are no longer students, so this finding is clearer.

Analysis of the average values of QLS scale scores in relation to the level of IQ, did not have statistically significantly different values of QLS scale on the first test, but after the one year, these QLS scale values increased statistically significantly in the subjects of the experimental group. In the control group, QLS values didn't increased significantly although their intellectual level was within the same category as that of the experimental group. In favor of this we suppose that intellectual abilities are a significant predictor of improving the quality of life only in those who are involved in socio-psychological rehabilitation treatment. In those psychotic disorders in which good intellectual potentials have been registered, but which are exclusively on psychopharmaceuticals, intellectual abilities will not produce significant changes in their quality of life. 
In the experimental group, the average value of the QLS scale after one year analyzed by the number of hospitalizations showed that the average value of the QLS scale was inversely correlated with the number of hospitalizations. Quality of life is therefore in subjects of experimental group directly connected with/ reduction/number of rehospitalizations.

The results of the control group of subjects, where that average value of the QLS scale was inversely correlated with the number of hospitalizations. The subjects of control group experienced that their quality of life has increased with more frequent hospitalizations, what's seemingly paradoxical. These findings introduce us to the following discussion: group psychotherapy in the experimental group of subjects leads to a significant improvement of the quality of life, and directly to the reduction of the number of psychiatric hospitalizations. If the subjects of the experimental group relapsed, rehospitalization directly affects the perception of lower quality of life.

The situation is completely reversed in the group of subjects of the control group. Namely the findings suggest that rehospitalization in their perception affects the improvement of their quality of life.Our question is, how to understand these opposites of such a different subjective perception of subjects belonging to the same category of mental disorders? The subjects of the experimental group have their group, as a "safe place" where they can feel intimacy and express their fears, feelings and fantasies. The group is both a "father and a mother and a brother and sister" and with its safe setting, provides a "safe" place and a stage for the most intense social interactions, for a long period of time to their members. A group is a place learn new models of adaptation to reality. They learn from each other, or like Bion said, they learn from experience (Bion,1984), They gain a feeling of self-worth in the group and during the time become respected by group members, gaining a certain position in the group that changes depending on the course of the group process. The subjects of the control group are lonely and most often socially isolated, often "cut off", from their family members, who often reject them.Psychotic subject without a group support, seeks protection in a hospital. It is not uncommon for some of subjects, who had developed the syndrome of hospitalism, to stop to use pharmacotherapy very intentionally, in order to come to their psychiatric ward again. Hospital treatment provides protection, safety and communication with others who are in the same position. It becomes a substitute for life, the kind of "womb" where theyhide and exist only in the role of "patient", but not in the other roles, that life in an open society requires. The hospital environment becomes a substitute for life and gives them a false experience of quality living, and in fact they are not able to get to know a different way of life. Hospital environment is better for them than home isolation and that is why they perceive it as quality, as well as their life in it, in relation to the subjects of the experimental group, for whom the group is a transitional object, on their way to real life, to the society in which they attend to live.

\section{CONCULSION}

Based on the results obtained through the present study, several conclusions can be derived there from:

1. On beginning of this study there are statistically significant results of the poor life quality of the both group of subjects: experimental and the control group.

2. In the experimental group, the average value of the QLS scale is statistically significantly higher on retesting compared to the same value of the QLS scale on the first test. In the control group of subjects there were no statistically significant differences in quality of life compared to the first test.

3. The existence of statistically significant difference between the experimental and control group according to different variables (sex, education, work activity, number of readmissions, day functionality, etc.) were established.

4. When we take into account the number of years spent in psychodrama group, we could see that with more years spent on group psychotherapy treatment, the score on the quality of life scale is higher. The best results are in those patients who spent 3-4 years in the group. In subjects who spent 5 or 6 years, in the group treatment, quality of life begins to decline.

The obtained results may only serve as a "pilot study" for understanding the impact of the psychodrama group treatment on quality of life of psychotic patients (schizophrenia spectar). These findings are the good starting argument for the integrative treatment of subjects who suffer from the psychoses.Most of our findings require additional verification that would be better methodologically designed. Also, the sample in this mini-research is very small and for a more valid conclusion it is necessary to have a larger sample. However, any more precise, conclusions regarding the influence of different variables (sex, education, work activity, number of readmissions, day functionality, etc.) on the life quality of psychotic subjects requires larger number of respondents and a larger number of random chosen respondents and more sophisticated levels of data analysis. In this respect we should point out that our findings on our quazi-experimental study are limited by the small a non-sufficiently representative sample. Further examinations of the Life Quality of psychotic subjects are needed, not only because of better research methodology, but because of better tertiary prevention of chronic psychotic illness. The basis of psychodrama group work is theinteraction. The psychodrama plays illuminates in the group member the interplay of intrapsychic and interpersonal, transforming invisible into visible. With the findings that we obtained in this pilot study, we must put us some questions: What is therapeutic agent in psychodrama which must effect on the alleviation, reduction of psychological suffering, to correct and modify the behavior and better reorganize the personality? The luck of valid and reliable methodology prevents us to answer on this question. 


\section{REFERENCES}

Barr, M.A. (1986). Homogeneous groups with acutely psychotic shizophrenics. Group, 10(1), 7-12.

Bellil, E. (2011). The effects of psychodrama on depression and mental state among women with chronic mental disorder. European Psychiatry, 25(1), 621. https:// www.sciencedirect.com/science/article/abs/pii/ S0924933811723276, seen 26.04.2021.

Bion, W. (1984). Iskustvo u radusagrupamaidrugiradovi. Psihologijailjudskadvojba. Naprijed. Zagreb.

Brkic, M., Tadic, M., Stojiljkovic, D., Maric, N. (2005).Sex differences and schizophrenia spectrum disorders. Engrami, 27(3-4), 13-19.

Eack, S.M., Newhill, C.E., Anderson, C.M., Rotondi, A.J. (2007). Quality of life for persons living with schizophrenia: more than just symptoms. PsychiatrRehabil J; 30(3), 219-22.

Foulkes, S.H.(1975). Group-Analytic Psychotherapy: Methods and Principles.London: Gordon \&Breach.

Heider, D., Angermeyer, M.C., Winkler, I., Schomerus, G., Bebbington, P.E. Brugha, T., et al. (2007). A prospective study of Quality of life in schizophrenia in three European countries. SchizophrRe, 93(1-3): 194-202.

Heinrichs, D.W., Hanlon, T.E., Carpenter, W.T.(1984). The Quality of LifeScale: One instrument for rating schizophrenic deficit syndrome. Shizophr. Bull,10(3), 388-98.

Herrman, H., Hawthorne, G., Thomas, R. (2002). Quality of life assessmentin people living with psychosis. Soc. Psychiatry Psychiatr. Epidemiol,37(11): 510-8.

Holzner, B., Kemmler, G., Meise, U. (1998).The impact of workrelated rehabilitation on the quality of life of patients with schizophrenia. Soc Psychiatry Psychiatr Epidemiol, 33(12): 624-31.

Lehman, A., Steinwachs, D., \& the Survey Co'Investigators of the PORT Project. (1998). Patterns of Usual Care for Schizophrenia:Initial Results From the Schizophrenia Patient Outcomes Research Team (PORT) Client Survey. Schizophrenia Bulletin, 24(1), 11-19.

Moreno, J.L.(1946). Psychodrama (Vol.I). New York: Beacon House

Ochoa, S,. Usall, J., Cobo, J., Labad, H.,\& Kulkami, J. (2012). Gender Differences in Schizophrenia and FirstEpisode Psychosis: A Comprehensive Literature Review. Shizophr.Res Treatment. 2012: 916198. doi: 10.1155/2012/916198, https://www.ncbi.nlm.nih. gov/pmc/articles/PMC3420456/, seen 30.04.2021.
Röder-Wanner, U.U., Oliver, J.P., Priebe, S. (1997). Does quality of life differin schizophrenic women and men? An empirical study. Int JSoc Psychiatry; 43(2), 129-43.

Rosenfeld, H. (1965). Psychotic States: A Psychoanalytic Approach. London: The Hogarth Press.

Ruzic, K., Medved, P., Dadic-Hero, E., Tomljanovic, D. (2009). Rehabilitation in psychaitry -sociotherapy. Medicine, 45(4), 338-343. (In Croatian)

Schmidt, K., Staupendahl, A., Vollmoeller, W. (2004). Quality of life ofschizophrenic psychiatric outpatients as a criterion for treatment planning in psychiatric institutions. Int $J$ Soc Psychiatry; 50(3): 262-73.

Sullivan, H.S.(1974). Shizophrenia as a human process. W.W Norton@Company. INC. New York.

Veljkovic, J. (2011). Follow-up study of the effects of psychodrama on patients suffering from psychosis. The British Journal of Psychodrama and Sociodrama, 12(1-2), 6-28.(In Serbian)

Veljkovic, J. (2014). Psychodrama changes. Andrejevic Endowment. Belgrade (In Serbian)

Veljkovic, J. (2015). Application of psychodrama in integration of self in adolescents. Sociology. LVII(2), 199-330. (In Serbian)

Veljkovic, J. (2019). How to fight against stigma and auto-stigma of persons diagnosed with psychosis? Presentation of the treatment of psychotic disorders in two social contexts. Sociologycal Review, LIII(4), 1553-1583. (In Serbian and English)

Veljkovic, J., \& Djuric, Z. (1997). Application of psychodrama in the treatment of psychotic patients. Engrami, 19(1-2), 95-105. (In Serbian)

Wieser, M. (2007). Studies on treatment effects of psychodrama psychotherapy.In:Psychodrama-Advances in Theory and Practise of Group Psychotherapy. Routledge: London, 271-293.

World Health Organization. (1992). The ICD-10 Classification of Mental and Behavioral Disorders - 1st.ed. Geneva: World Health Organization. 